\title{
Changes in testosterone levels and sex hormone-binding globulin levels in extremely obese men after bariatric surgery
}

Patchaya Boonchaya-anant 1,2, Nitchakarn Laichuthai ${ }^{1,2}$, Preaw Suwannasrisuk ${ }^{2,3}$, Natnicha Houngagam ${ }^{1,2}$, Thiti Snabboon $^{1,2}$ ${ }^{1}$ Hormonal and Metabolic Disorders Research Unit, Excellence Center for Diabetes, Hormone, and Metabolism, Department of Medicine, Faculty of Medicine, Chulalongkorn University 2 Division of Endocrinology and Metabolism, Department of Medicine, Faculty of Medicine, Chulalongkorn University, and King Chulalongkorn Memorial Hospital, Thai Red Cross Society ${ }^{3}$ Department of Medicine, Faculty of Medicine, Naresuan Univerisity Hospital, Phitsanulok, Thailand

\section{Objectives}

- Obesity is a risk factor for hypogonadotrophic hypogonadism in men. Increase in body weight was found to be associated with decrease testosterone level and sex hormone-binding globulin (SHBG) level.

- The aim of this study was to evaluate the change in testosterone levels in extremely obese men after bariatric surgery.

\section{Methods}

This is a prospective study including 29 morbidly obese men undergoing bariatric surgery.

- Main outcomes were changes in serum levels of total testosterone (TT), free testosterone (cFT), SHBG, Estradiol, adiponectin and leptin at 1 and 6 months after bariatric surgery.

\begin{tabular}{|c|c|}
\hline Age (year) & $30.8 \pm 8.1$ \\
\hline \multicolumn{2}{|l|}{ Comorbidities [n(\%)] } \\
\hline Hypertension & 19 (65.5\%) \\
\hline Diabetes & 7 (24.1\%) \\
\hline Dyslipidemia & $19(65.5 \%)$ \\
\hline NAFLD/NASH & 20 (69.0\%) \\
\hline Obstructive sleep apnea & $29(100 \%)$ \\
\hline \multicolumn{2}{|l|}{ Type of surgery } \\
\hline RYGB & $15(51.7 \%)$ \\
\hline SG & $14(48.3 \%)$ \\
\hline Body weight (kg) & $168.3 \pm 35.0$ \\
\hline BMI $\left(\mathrm{kg} / \mathrm{m}^{2}\right)$ & $56.8 \pm 11.7$ \\
\hline SBP (mmHg) & $133 \pm 26$ \\
\hline DBP (mmHg) & $83 \pm 12$ \\
\hline FPG (mg/dL) & $99.0(89.3-122.8)^{*}$ \\
\hline HbA1c (\%) & $6.0(5.2-6.3)^{*}$ \\
\hline Total Cholesterol (mg/dL) & $211 \pm 56$ \\
\hline HDL (mg/dL) & $39 \pm 11$ \\
\hline Triglyceride (mg/dL) & $178 \pm 86$ \\
\hline LDL (mg/dL) & $139 \pm 42$ \\
\hline $25-\mathrm{OH}$ vitamin $\mathrm{D}(\mathrm{ng} / \mathrm{ml})$ & $18.2 \pm 5.5$ \\
\hline
\end{tabular}

Data are expressed as means + SD or number (percentage)

*Median (Interquartile range).

\section{Results}

- Sixteen patients underwent Roux-en- $Y$ gastric bypass and 13 patients underwent sleeve gastrectomy.

- At baseline, 22 patients (75.9\%) had low TT levels (<10.4 nmole/L) and 16 patients (55.2\%) had low cFT levels (<225 pmole/L).

- Total testosterone and SHBG levels increased significantly at 1 month after surgery $(p=<0.001)$ whereas cFT levels have not changed.

- At 6 months after surgery, TT, cFT and SHBG levels increased significantly (all $p$-values $=<0.001$ ) and 23 patients $(79.3 \%$ ) had normal TT levels.

- There were no changes in estradiol levels.

- Leptin levels decreased and adiponectin levels increased significantly after surgery.

- Early increases in TT levels were associated with increases in SHBG levels ( $r=0.472, p=0.042$ ) but not with changes in body weight, BMI, adiponectin or leptin.

\begin{tabular}{|c|c|c|c|}
\hline & Baseline & Month 1 & Month 6 \\
\hline Body weight (kg) & $168.3 \pm 34.9$ & $146.0 \pm 31.7^{b}$ & $126.2 \pm 25.5^{b}$ \\
\hline BMI $\left(\mathrm{kg} / \mathrm{m}^{2}\right)$ & $56.9 \pm 11.7$ & $50.2 \pm 11.0^{b}$ & $42.9 \pm 9.0^{b}$ \\
\hline $\begin{array}{l}\text { Total testosterone } \\
\text { (nmole/L) }\end{array}$ & $8.38 \pm 4.67$ & $12.60 \pm 6.09 b$ & $15.81 \pm 5.95^{b}$ \\
\hline $\begin{array}{l}\text { Calculated free } \\
\text { testosterone } \\
\text { (pmole/L) }\end{array}$ & $214.0 \pm 113.0$ & $245.3 \pm 114.7$ & $307.9 \pm 121.7^{b}$ \\
\hline SHBG (nmole/L) & $21.59 \pm 10.71$ & $39.59 \pm 21.46^{b}$ & $40.67 \pm 15.48^{b}$ \\
\hline $\begin{array}{l}\text { Estradiol } \\
\text { (pmole/L) }\end{array}$ & $155.1 \pm 56.6$ & $155.2 \pm 39.4$ & $149.5 \pm 47.1$ \\
\hline $\begin{array}{l}\text { Adiponectin } \\
(\mu \mathrm{g} / \mathrm{ml})\end{array}$ & $6851.5 \pm 4793.5$ & $11942.9 \pm 8727.6^{b}$ & $12456.2 \pm 9160.2^{b}$ \\
\hline Leptin (ng/ml) & $46.21 \pm 18.86$ & $32.84 \pm 17.72^{b}$ & $21.32 \pm 11.01^{b}$ \\
\hline
\end{tabular}

Compared with baseline values using paired t-tests; ${ }^{\mathrm{a}} \mathrm{p}<0.05,{ }^{\mathrm{b}} \mathrm{p}<0.01$

Figure 1. Changes in parameters after bariatric surgery

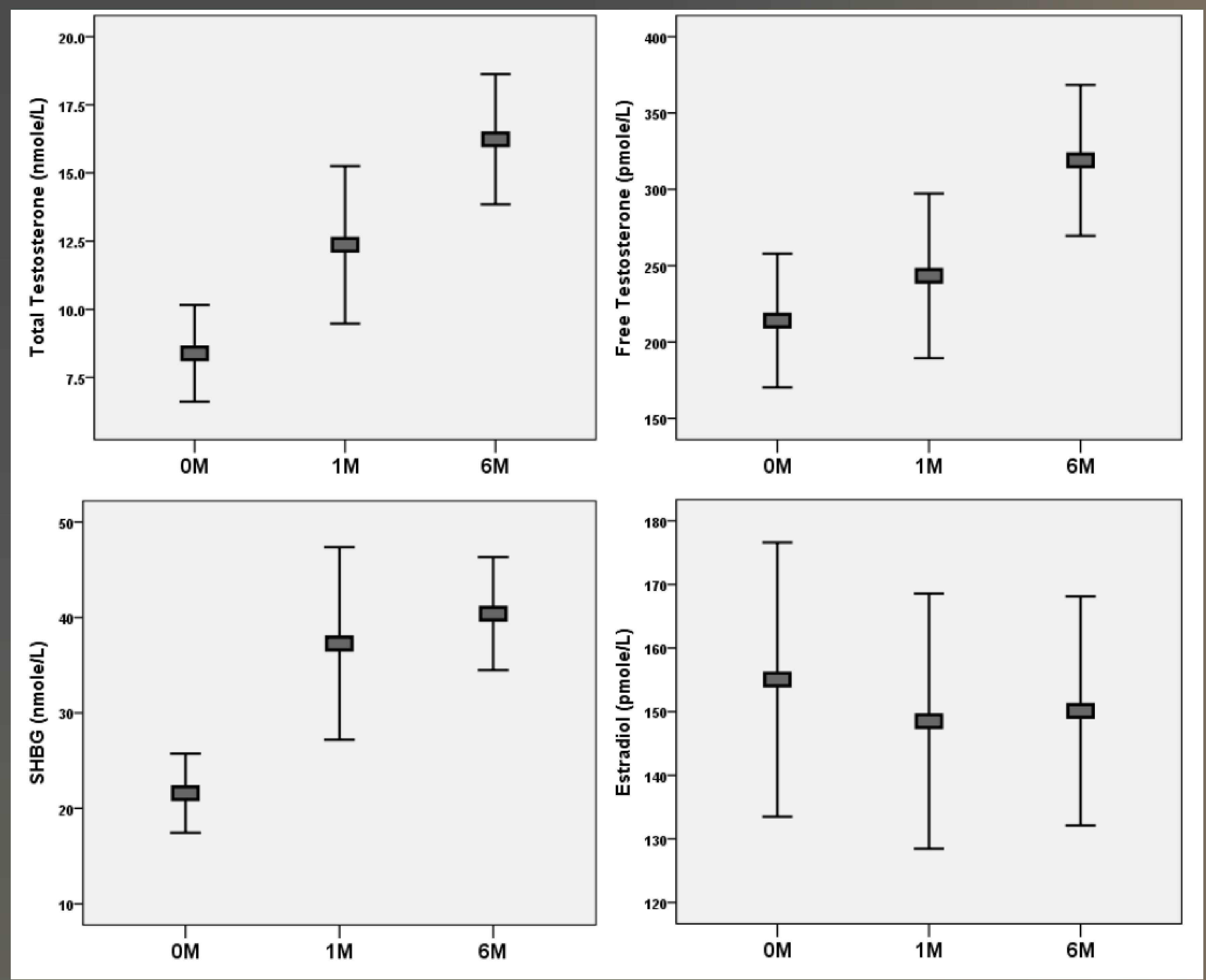

\section{Conclusion}

Increases in TT and SHBG levels occurred early at 1 month after bariatric surgery while improvements in cFT levels were observed at 6 months after bariatric surgery.

- No change in estradiol levels was found.

- Changes in total testosterone levels tend to be correlated with SHBG levels but not with body weight or adipokines levels.

\section{References}

1. Hammoud A, Gibson M, Hunt SC, et al. Effect of Roux-en-Y gastric bypass surgery on the sex steroids and quality of life in obese men. The Journal of clinical endocrinology and metabolism. 2009;94(4):1329-32.

2. Woodard G, Ahmed S, Podelski V, et al. Effect of Roux-en-Y gastric bypass on testosterone and prostate-specific antigen. The British journal of surgery. 2012;99(5):693-8.

3. Botella-Carretero JI, Balsa JA, Gomez-Martin JM, et al. Circulating free testosterone in obese men after bariatric surgery increases in parallel with insulin sensitivity. Journal of endocrinological investigation. 2013;36(4):227-32. 